\title{
Simultaneous imaging and diffraction of phase transitions at intermediate compression rates
}

\author{
R. J. Husband ${ }^{1}$, Zs. Jenei ${ }^{2}$, J. Hagemann'1, E. F. O'bannon², W. J. Evans ${ }^{2}$, A. Schropp ${ }^{1}$, K. Glazyrin ${ }^{1}$ and \\ H. P. Liermann ${ }^{1}$ \\ ${ }^{1}$ DESY, Notkestrasse 85, 2260 Hamburg, Germany \\ ${ }^{2}$ Lawrence Livermore National Laboratory, 7000 East Avenue, L-041 Livermore, CA 94550, USA \\ rachel.husband@desy.de
}

Fast compression in the dynamic diamond anvil cell (dDAC) allows for the study of materials at intermediate strain rates that are not accessible using traditional static and dynamic compression techniques [1]. Previous dDAC studies revealed compression-rate dependent phenomena such as rate-dependent phase transformation pathways [2], the formation of metastable phases [3], and shifts in phase transition boundaries from their equilibrium positions $[2,3,4]$. The fast diffraction set-up at the Extreme Conditions Beamline (P02.2) at PETRA-III offers time-resolved X-ray diffraction with $\mathrm{kHz}$ data collection rates, which allows for phase transition boundaries to be accurately determined at compression rates up to $\sim 1000 \mathrm{GPa} / \mathrm{s}$. Future experiments at the European XFEL will allow for data collection rates up to $4.5 \mathrm{MHz}$, which will extend these studies to compression rates $>100 \mathrm{TPa} / \mathrm{s}$.

In order to develop a full understanding of phase transitions under dynamic compression, it is necessary to investigate sample behaviour on both atomistic (crystal structure) and microscopic (crystal morphology) length scales. This allows for kinetic parameters such as nucleation and growth rates to be determined. When crystallite of the high-pressure phase have well-defined phase boundaries, imaging techniques can be used to visualize the growth of the new phase. The X-ray phase contrast imaging platform at P02.2 allows for the visualization of samples that are opaque to visible light, where the simultaneous X-ray diffraction measurements allow for pressure determination, phase identification, and structural refinement. Phase contrast imaging allows us to resolve phase boundaries for grains of similar Z, where conventional absorption-based imaging typically fails.

Here, we present results from X-ray imaging experiments on dynamically compressed Ga (Fig. 1), where we have successfully imaged pressure-induced melting (Ga-I/liquid) and solidification (liquid/Ga-III). Using an imaging configuration in which the sample is positioned upstream from the focal spot of a CRL-focussed X-ray beam allows for the collection of 'clean' diffraction patterns with minimal contribution from the gasket material and produces clearly-defined solid/liquid phase boundaries in the X-ray images.

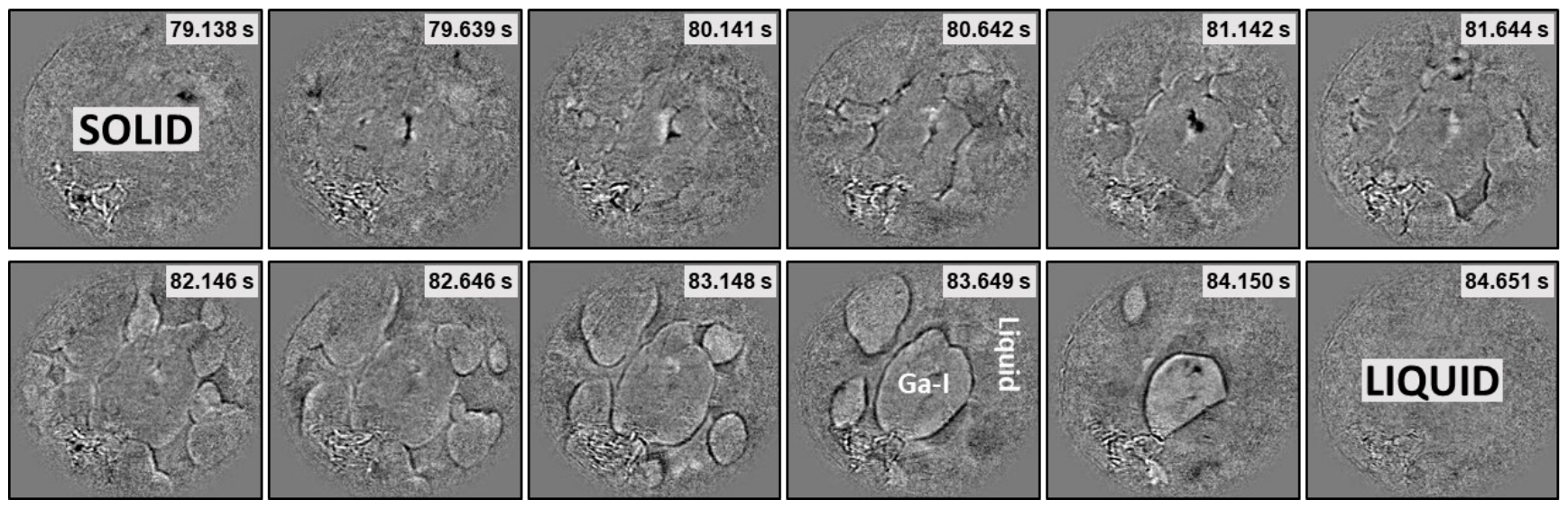

Figure 1. X-ray images showing the melting of Ga on compression in the dynamic-DAC

[1] Jenei, Zs. et al. Rev. Sci. Instrum. 90, 065114 (2019).

[2] Lee, G. W., Evans, W. J. \& Yoo, C. S. Phys. Rev. B 74, 134112 (2006).

[3] Chen, J. Y \& Yoo, C. S. PNAS 108 7685-7688 (2011).

[4] Husband. R. J. et al. 'Compression-rate dependence of pressure-induced phase transitions in Bi', submitted.

Keywords: Dynamic compression, diamond anvil cell, X-ray imaging 